4

5

6

7

8

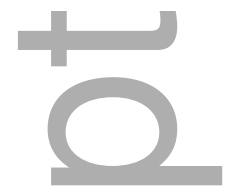

Article type : :Technical Notes

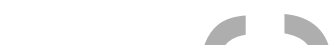

\title{
A method for fatigue testing of equine MCIII subchondral bone under a simulated fast workout training program
}

Shaktivesh ${ }^{1}$, F. Malekipour ${ }^{1}$, R. C. Whitton ${ }^{2}$ and P. V. S. Lee*1

${ }^{1}$ Department of Biomedical Engineering, University of Melbourne, Parkville, Victoria, 3010, Australia and ${ }^{2}$ Equine Centre, Melbourne Veterinary School, Faculty of Veterinary and Agricultural Sciences, The University of Melbourne, Werribee, Victoria, 3030, Australia.

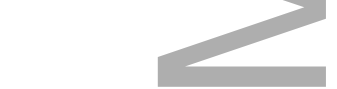

*Corresponding author email: pvlee@unimelb.edu.au

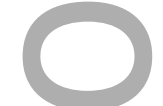

Keywords: horse; MCIII subchondral bone; fatigue life; training; racehorse; gaits

Running head: MCIII subchondral bone under a simulated fast workout.

\section{Summary}

Background: Standard fatigue testing of bone utilises a single load and frequency applied until failure. However, in situ, the subchondral bone of Thoroughbred racehorses is subjected to a combination (or a spectrum) of loads and frequencies during training and racing.

This is the author manuscript accepted for publication and has undergone full peer review but has not been through the copyediting, typesetting, pagination and proofreading process, which may lead to differences between this version and the Version of Record. Please cite this article as doi: $10.1111 / \mathrm{EVJ} .13163$

This article is protected by copyright. All rights reserved 
Objective: To investigate the use of a fatigue testing method for equine third metacarpal (MCIII) subchondral bone under a spectrum of loading conditions which a racehorse is likely to experience during a fast workout.

Study design: In vitro biomechanical experimental study.

Methods: MCIII subchondral bone specimens $(n=12)$ of racehorses were harvested from left and right medial condyles. A novel fatigue loading protocol was developed based upon a standard sequence of gaits during a typical fast workout protocol. This loading pattern, or loading loop, was repeated until the failure of the specimen.

Results: The mean \pm standard deviation for all specimens for total time-to-failure was 76,393 $\pm 64,243$ seconds (equivalent to $18.3 \pm 15.7$ fast workouts). Ten out of twelve specimens withstood at least one complete loop equivalent to a fast workout. All specimens failed during simulated gallop loading.

Main limitations: The resting time between loops was much shorter than in vivo resting time and specimens were unconfined during compressive testing.

Conclusions: This novel fatigue loading protocol more closely mimics in vivo fatigue loading of MCIII subchondral bone and demonstrates the importance of the highest speeds in the development of subchondral bone injury.

\section{Introduction}

Subchondral bone injuries and fractures commonly occur in the third metacarpal bone (MCIII) of Thoroughbred racehorses [1,2]. Such injuries have been associated with the number and frequency of galloping sessions and are therefore considered a result of bone material fatigue $[3,4]$. Thus, understanding the fatigue behaviour of subchondral bone in conditions similar to that experienced during racing or training could help minimising injuries. Previous fatigue studies of equine bone have applied a constant stress amplitude (varying sinusoidally) and frequency [5]. These studies involve establishing an S-N curve, where ' $\mathrm{S}$ ' is the stress in bone and ' $\mathrm{N}$ ' is the number of cycles-to-failure $[2,5]$. The relationship between the applied load and fatigue life follows a power law with a shorter fatigue life observed at higher loads [5]. However, training of racehorses results in varying joint reaction forces at varying stride frequencies within a single workout $[6,7]$.

To estimate damage due to fatigue under variable-amplitude loading PalmgrenMiner's rule is used. It is a linear damage accumulation model that uses the S-N curve as the input to determine cumulative damage based on a variable-amplitude load-time history [8]. 
However, Palmgren-Miner's rule has limitations such as assuming a linear accumulation of damage, not accounting for the interaction between varying stress levels and the probabilistic nature of fatigue. It has been shown that similar structures or specimens subjected to identical cyclic loading conditions display large variations [9]. To overcome such limitations, researchers have utilised alternative methods. For instance, in some aerospace applications, the fatigue life of aircraft material is evaluated by mimicking the loading conditions as experienced during a typical flight [10]. The application of realistic loading experienced by racehorses to study the fatigue properties of equine MCIII subchondral bone explants has not been implemented.

The load history of a typical racehorse fast workout session was applied to MCIII subchondral bone specimens to investigate their fatigue performance. The load history was simplified by a series of loadings known as spectrum loads simulating the load magnitude and frequency during walking, trotting, cantering, and slow and fast galloping. We expected that fatigue failure would occur predominantly during loading equivalent to the most intense gait (galloping), due to the power law relationship between applied load and fatigue life [5].

\section{Materials and Methods}

\section{Specimen preparation}

Subchondral bone specimens from the palmar aspect of condyles were harvested from left and right third metacarpal bones of Thoroughbred racehorses within 24 hours of death or euthanasia and stored at $-20^{\circ} \mathrm{C}$ wrapped in saline soaked gauze with marrow intact. Eight horses died or were euthanased for conditions other than a musculoskeletal injury, and four horses were euthanased for fractures of a limb other than the limb from which the sample was obtained. Twelve specimens were from horses in three age groups (2 years $[n=7], 3$ years [n $=4]$, and 4 years $[\mathrm{n}=1]$ ). The bone cores were collected 3-5 mm palmar to the transverse ridge, from the middle third of the condyle. A diamond coated core drill bit ${ }^{\text {a }}$ was used to extract cylindrical specimens from medial condyles perpendicular to the articular surface with a diameter of $6.50 \pm 0.07 \mathrm{~mm}$ and length of $6.81 \pm 0.57 \mathrm{~mm}$ (mean \pm s.d.).

\section{Experimental set-up}

A $\pm 10 \mathrm{kN}$ load-cell (Dynacell) ${ }^{\mathrm{b}}$ was fixed to the crosshead of a mechanical testing machine (Instron 8874) ${ }^{\mathrm{b}}$. A stainless-steel impactor was connected to an upper platen to load the specimen in unconfined compression (Fig 1). A chamber was fabricated using acrylic 
plastic and fixed on the base of the lower platen to contain saline solution $(0.9 \%$ sodium chloride solution) to maintain specimen hydration throughout the test. The test specimen was prepared by fixing it to the lower platen with cyanoacrylate [11] and left to cure for $5 \mathrm{~min}$ while wrapped in moist gauzes. The lower platen along with the saline-filled chamber was placed on the cylindrical plate (Fig 1).

\section{Mechanical Loading}

Wavematrix software (version 1.4.268.0) $)^{\mathrm{b}}$, installed on a computer system which interfaced with the mechanical testing machine, was used to control the input signal and read the output data such as crosshead position and force. Each specimen was first preconditioned by applying 50 cycles of compressive stress at $20 \mathrm{MPa}$ at $2 \mathrm{~Hz}$, which is $18 \%$ of the compressive yield stress (113.3 MPa) reported for the lateral condyle of MCIII [12]. Based on previous studies, such precondition loads did not cause any damage to the specimens $[5,13]$.

\section{$[5,13]$}

Fatigue loads were applied using a waveform signal block loading (defined as one training loop). Each training loop comprised a succession of compression-compression (c-c) sinusoidal loading cycles with varying load-amplitudes and frequencies based on published data for four gaits and five speeds: walk, trot, canter, slow gallop and fast gallop $[6,7,14]$ (Table 1) (Fig 2A and 2B). To determine the number of loading cycles, the distances covered during a fast workout under each gait or speed (obtained from training data collected for another study) were converted into the number of loading cycles based on the number of estimated ground strikes (Table 1) [14]. The estimated ground strikes for each gait were further divided equally into warm-up and cool-down stages. One load cycle was considered equivalent to one stance phase of the gait. The rest period between workouts was represented by a constant $15 \mathrm{MPa}$ pressure for $10 \mathrm{~min}$. The waveform block was applied repeatedly until the specimen failed. Fatigue failure was defined as a $10 \%$ reduction in the initial Young's modulus [13]. A predetermined safety limit (10\% of specimen's height) was set to stop the motion of the crosshead in case of gross failure of the specimen.

Total number of cycles-to-failure (TCF) and total time-to-failure (TTF) were specified as the total number of cycles and total amount of time respectively until a specimen failed. Gait specific time (GST (\%)), was defined as: 
Gait Specific Time, GST $(\%)=\frac{\text { Cumulative time spent for a specific gait until failure }}{\text { Total time to failure }(\text { TTF })} \times 100$

The calculations of mean and s.d. were performed in MATLAB ${ }^{c}$ and Excel ${ }^{d}$. Minitab18 $8^{\mathrm{e}}$ was used to test the results for normality using the Anderson-Darling method.

\section{Results}


Ten out of twelve specimens were able to withstand all the stress levels which were included in a training loop at least once (one fast workout session). This result was expected as the maximum stress amplitude remained below $80 \%$ of the yield strength of MCIII subchondral bone [12].

Even though specimens underwent loads representative of slower speed gaits for the greatest proportion of time, all specimens failed under simulated gallop loading (Table 2). Based on previously published fatigue data the proportion of specimen fatigue life expended during loading that simulated galloping in each training loop was six times that of all other simulated gait loading combined [5]. Therefore, we would expect most of our samples to fail in gallop as observed (Table 1). Additionally, equine MCIII subchondral bone fatigue life under a constant stress-amplitude of $90 \mathrm{MPa}$ was previously reported at (mean, median, range) 4,486, 4,001, 152-11,568 cycles [5] and we found that cumulative gallop specific loading cycles were 3,591 $\pm 3,030$ cycles, which is a good match. Even though all specimens were tested under similar loading conditions, the large variation in their fatigue life is likely due to their differing morphology and density [19]. Future studies will include the effects of bone morphology on the fatigue performance.

We utilised a rest period within the loading protocol which was shorter than that which occurs in vivo between workouts however there is no evidence that longer rest periods affect bone properties [20]. During compression tests, it is difficult to accurately mimic the degree of constraint conferred by neighbouring bone tissue. In our study, the bone specimens were loaded in an unconfined manner which is a recommended method for measuring mechanical properties of bone and to facilitate comparison with existing literature [21].

\section{Conclusion}

The fatigue properties of subchondral bone can be investigated using a fatigue testing protocol that includes a spectrum of loads applied to a single specimen similar to those experienced in vivo in a racehorse in training. We found that all the specimens failed under load equivalent to galloping which is consistent with what happens in vivo.

\section{Authors' declarations of interests}

No competing interests have been declared. 


\section{Ethical animal research}

195 The use of animal tissues met the requirements of the University of Melbourne Animal Ethics 196 Committee ID:14001.

197

\section{Owner informed consent}

199 Owners gave informed consent for their horses' inclusion in this study.

200

201

Source of funding

202 This study was funded by Racing Victoria Limited, the Victorian Racing Industry Fund of the 203 Victorian State Government and Melbourne Research Scholarship awarded by The 204 University of Melbourne.

205

206

Authorship

207 Shaktivesh andF. Malekipour contributed to study design, study execution, data analysis and 208 interpretation, and preparation of the manuscript. C. Whitton and P. Lee contributed to study 209 design, study execution, interpretation, and preparation of the manuscript. All authors gave 210 their final approval of the manuscript.

\section{Manufacturers' addresses}

213 aStarlite Industries Inc., Rosemont, Pennsylvania, USA.

214 bInstron

215

'Mathworks

216 dicrosoft, Redmond, Washington, USA.

217

eMinitab 
221 Table 1: Estimated equivalent values for forelimb ground strikes*, loading cycles, contact 222 pressure, and stride frequencies for each gait of a fast workout of a race fit adult 223 Thoroughbred racehorse [14,15-17]

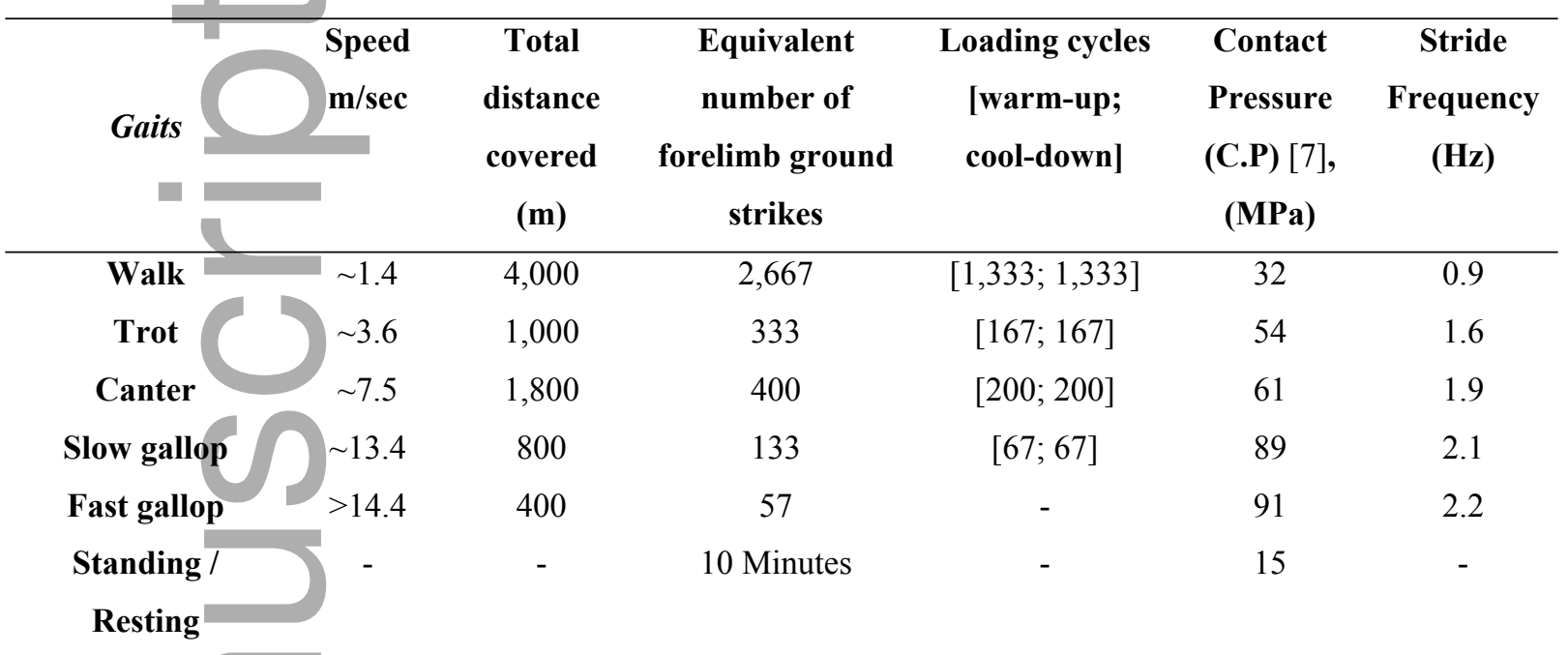

224

*Assuming the stride lengths are $7 \mathrm{~m}, 6 \mathrm{~m}, 4.5 \mathrm{~m}, 3 \mathrm{~m}, 1.5 \mathrm{~m}$ for different gaits at speeds of $14.4 \mathrm{~m} / \mathrm{s}, 13.4 \mathrm{~m} / \mathrm{s}, 7.5 \mathrm{~m} / \mathrm{s}, 3.6 \mathrm{~m} / \mathrm{s}, 1.4 \mathrm{~m} / \mathrm{s}$ respectively $[14,15,18]$

Table 2: GST \% (Gait Specific time) (Averaged for all specimens).

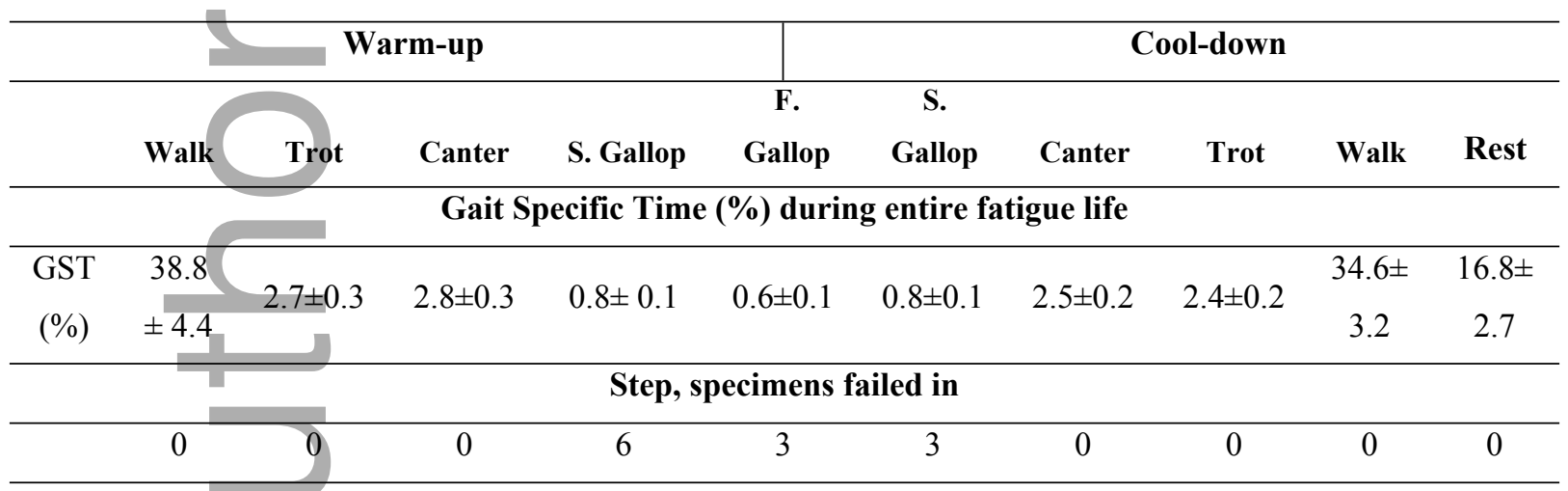

\section{Figure legends}

Fig 1: Experimental set-up for fatigue tests. 
Fig 2: A) A waveform signal indicating the repetition of a training loop (1-day fast workout).

B) The loading cycles within a simulated training loop, including a warm-up and cool-down routine, each warm-up and cool-down routine included cyclic loading that simulated loading conditions during walk, trot, canter, slow gallop and fast gallop of a racing horse. The frequency and load of each loading gait type is listed in Table 1.

\section{References}

1. Weill, J., Benaïm, Balle, Le and Barbe (1965) Syndrome de Morgagni-Adams-Stokes révélateur d'une maladie de Bouillaud. Bull. Mem. Soc. Med. Hop. Paris 116, 729-740.

2. Martig, S., Chen, W., Lee, P.V.S. and Whitton, R.C. (2014) Bone fatigue and its implications for injuries in racehorses. Equine Vet. J. 46, 408-415.

3. Verheyen, K., Price, J., Lanyon, L. and Wood, J. (2006) Exercise distance and speed

4. Pinchbeck, G.L., Clegg, P.D., Boyde, A. and Riggs, C.M. (2013) Pathological and clinical features associated with palmar/plantar osteochondral disease of the metacarpo/metatarsophalangeal joint in Thoroughbred racehorses. Equine Vet. J. 45, $587-592$

5. Martig, S., Lee, P.V.S., Anderson, G.A. and Whitton, R.C. (2013) Compressive fatigue life of subchondral bone of the metacarpal condyle in thoroughbred racehorses. Bone 57, 392-398.

6. Seder, J. and Vickery, C.E. (2003) Temporal and Kinematic Gait Variables of Thoroughbred Racehorses At or Near Racing Speeds. J. Equine Vet. Sci. III, 82-112.

7. Harrison, S.M., Whitton, R.C., Kawcak, C.E., Stover, S.M. and Pandy, M.G. (2014) Evaluation of a subject-specific finite-element model of the equine metacarpophalangeal joint under physiological load. J. Biomech. 47, 65-73.

8. Suresh, S. (1998) Fatigue of Materials 2nd Edn. Cambridge University Press.

9. Blason, S., Correia, J.A.F.O., Jesus, A.M.P. De, Calcada, R.A.B. and FernandezCanteli, A. (2016) A probabilistic analysis of Miner's law for different loading conditions. Struct. Eng. Mech. 60, 71-90. 
10. Schjive, J. (2003) Fatigue of Structures and Materials in the 20th Century and the State of the Art. Int. J. Fatigue 25, 679-702.

11. Athanasiou, K.A., Agarwal, A. and Dzida, F.J. (1994) Comparative study of the intrinsic mechanical properties of the human acetabular and femoral head cartilage. $J$. Orthop. Res. 12, 340-349.

12. Rubio-Martínez, L.M., Cruz, A.M., Gordon, K. and Hurtig, M.B. (2008) Mechanical properties of subchondral bone in the distal aspect of third metacarpal bones from Thoroughbred racehorses. Am. J. Vet. Res. 69, 1423-1433.

13. Moore, T.L.A. and Gibson, L.J. (2003) Fatigue of Bovine Trabecular Bone. J. Biomech. Eng. 125, 761-768.

14. Witte, T.H., Hirst, C. V. and Wilson, A.M. (2006) Effect of speed on stride parameters in racehorses at gallop in field conditions. J. Exp. Biol. 209, 4389-4397.

15. Yamanobe, A., Hiraga, A. and Kubo, K. (1992) Relationships between stride frequency, stride length, step length and velocity with asymmetric gaits in the thoroughbred horse. Japanese J. Equine Sci. 3, 143-148.

16. Nankervis, K., Marlin, D. and Hodgins, D. (2007) Comparison of stride frequency measured using a leg mounted accelerometer and opto-electronic system. Business 5151.

17. Ratzlaff, M.H. (1989) Quantitative methods for the analysis of equine locomotion and their applications to other species. Integr. Comp. Biol. 29, 267-285.

18. Hodson, E., Clayton, H.M. and Lanovaz, J.L. (2000) The forelimb in walking horses: 1. Kinematics and ground reaction forces. Equine Vet. J. 32, 287-294.

19. Carter, D.R., Caler, W.E., Spengler, D.M. and Frankel, V.H. (1981) Uniaxial fatigue of human cortical bone. The influence of tissue physical characteristics. J. Biomech. 14, $461-470$.

20. Holmes, J.M., Mirams, M., Mackie, E.J. and Whitton, R.C. (2014) Thoroughbred horses in race training have lower levels of subchondral bone remodelling in highly loaded regions of the distal metacarpus compared to horses resting from training. Vet. J. 202, 443-447. 

(2018) Standardizing compression testing for measuring the stiffness of human bone. Bone Joint Res. 7, 524-538.

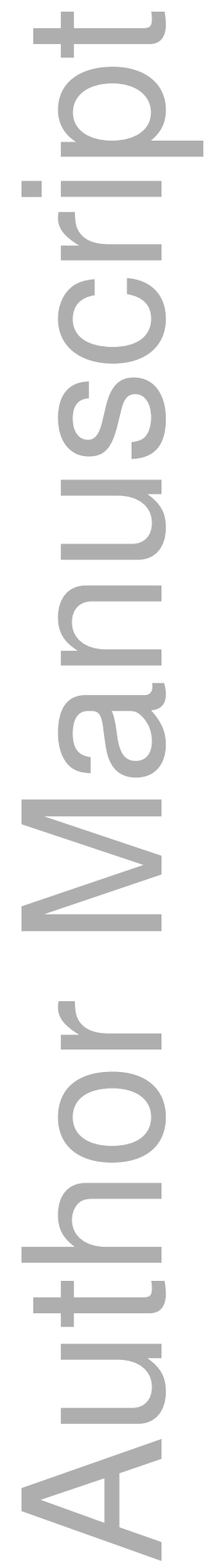




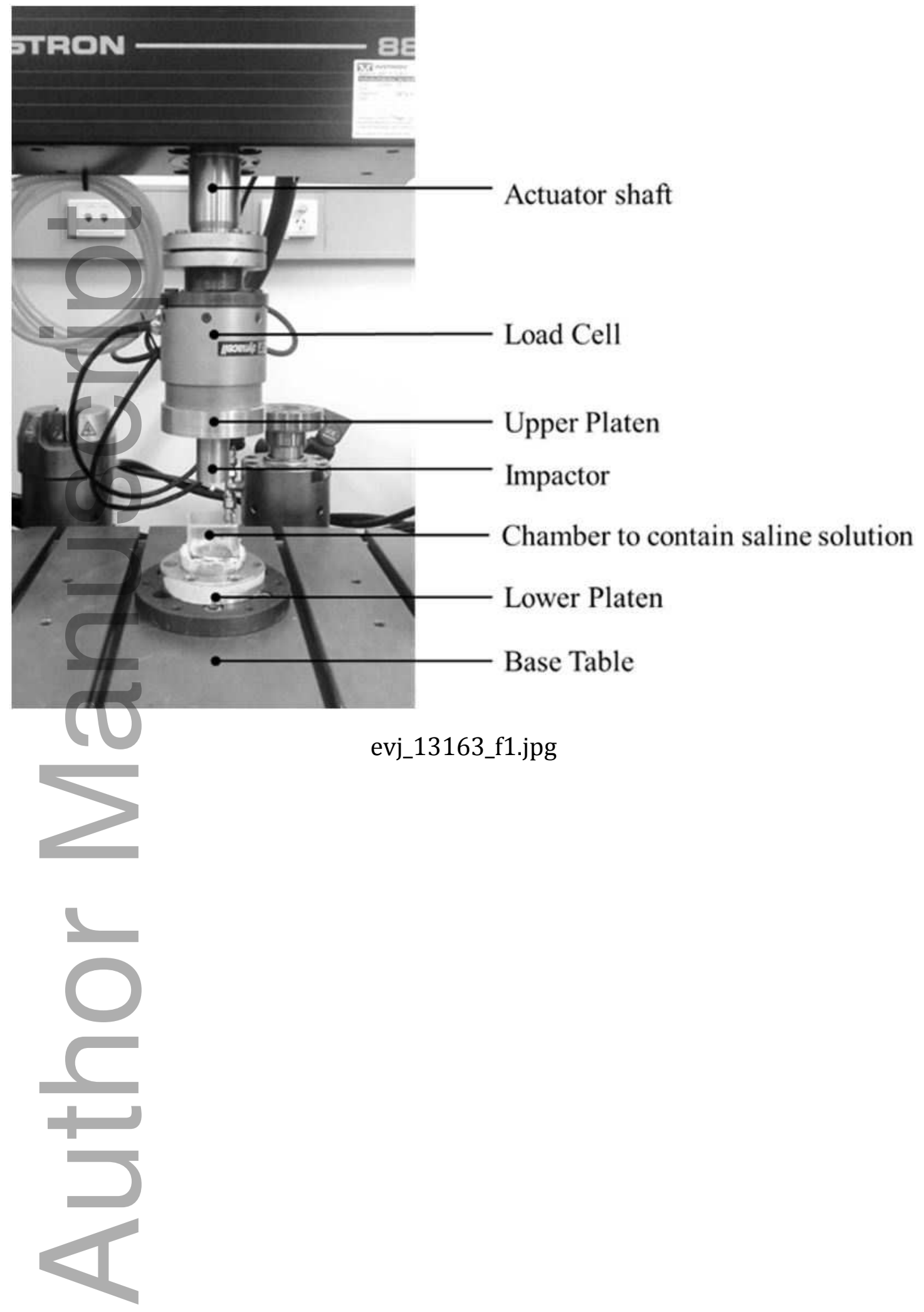

This article is protected by copyright. All rights reserved 
A. Waveform Signel o: fat.gue loedice io rapast a irsiuing boy
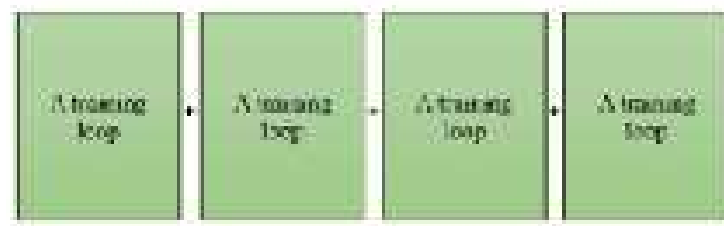

(B)

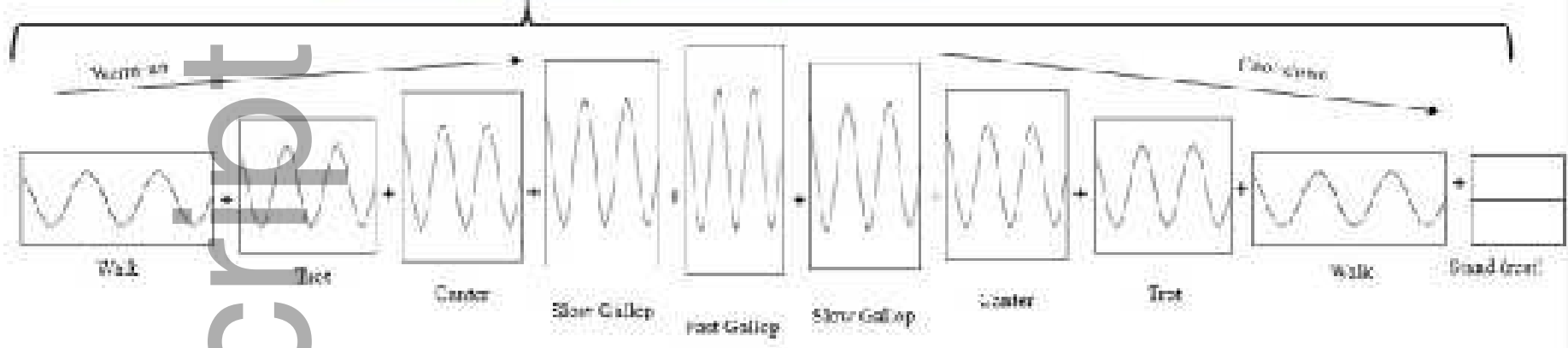

S

evj_13163_f2.jpg
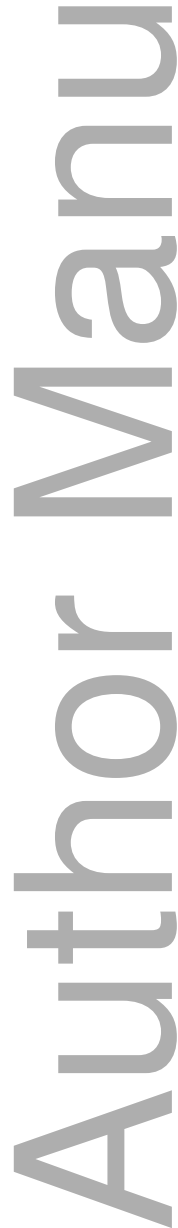


\section{University Library}

\section{- M M N E R VA A gateway to Melbourne's research publications}

Minerva Access is the Institutional Repository of The University of Melbourne

\section{Author/s:}

Shaktivesh,;Malekipour, F;Whitton, C;Lee, PVS

Title:

A method for fatigue testing of equine Mclll subchondral bone under a simulated fast workout training programme

\section{Date:}

2020-03-01

\section{Citation:}

Shaktivesh, , Malekipour, F., Whitton, C. \& Lee, P. V. S. (2020). A method for fatigue testing of equine Mclll subchondral bone under a simulated fast workout training programme. Equine Veterinary Journal, 52 (2), pp.332-335. https://doi.org/10.1111/evj.13163.

Persistent Link:

http://hdl.handle.net/11343/286404 